\title{
Constraining positron emission from pulsar populations with AMS-02 data
}

\author{
Luca Orusa, ${ }^{a, b, *}$ Silvia Manconi, ${ }^{c}$ Mattia Di Mauro ${ }^{b}$ and Fiorenza Donato ${ }^{a, b}$ \\ ${ }^{a}$ Dipartimento di Fisica, Università di Torino, \\ Via P. Giuria 1, Torino, Italy \\ ${ }^{b}$ Istituto Nazionale di Fisica Nucleare, Sezione di Torino, \\ Via P. Giuria 1, 10125 Torino, Italy \\ c 2Institute for Theoretical Particle Physics and Cosmology, RWTH Aachen University, \\ Sommerfeldstr. 16, 52056 Aachen, Germany \\ E-mail: luca.orusa@edu.unito.it
}

The cosmic-ray flux of positrons is measured with high precision by the space-borne particle spectrometer AMS-02. The hypothesis that pulsar wind nebulae (PWNe) can significantly contribute to the excess of the positron $\left(e^{+}\right)$cosmic-ray flux has been consolidated after the observation of a $\gamma$-ray emission at $\mathrm{TeV}$ energies of a few degree size around Geminga and Monogem PWNe. In this work we simulate Galactic pulsars populations adopting different distributions for their position in the Galaxy as well as their intrinsic physical properties, in order to overcome the incompleteness of the ATNF catalogue. We fit the $e^{+}$AMS-02 data together with a secondary component due to collisions of primary cosmic rays with the interstellar medium. We find that several mock galaxies have a pulsar population able to explain the observed $e^{+}$flux, characherized typically by few, bright sources. We determine the physical parameters of the sources dominating the $e^{+}$flux, and assess the impact of different assumptions on radial distributions, spin-down properties and propagation scenarios.

$37^{\text {th }}$ International Cosmic Ray Conference (ICRC 2021)

July 12 th - 23rd, 2021

Online - Berlin, Germany

\footnotetext{
${ }^{*}$ Presenter
} 


\section{Introduction}

The observation of high-energetic cosmic-ray positrons with unprecedented precision by AMS02 [1] suggests the presence of primary positron $\left(e^{+}\right)$sources in our Galaxy, as the observed flux exceeds the so-called secondary flux produced by inelastic collisions of cosmic-ray nuclei in the interstellar medium above about $10 \mathrm{GeV}$. Pulsars have been consolidating as significant factories of high-energy cosmic-ray electrons and positrons $\left(e^{ \pm}\right)$in the Galaxy, and thus as main candidates to explain the $e^{+}$excess [2]. The observation of $\gamma$-ray halos at TeV energies of a few degree size around two nearby pulsars (Geminga and Monogem see [3]) corroborates the presence of $e^{ \pm}$ accelerated, and escaped, by their PWNe since the observed emission is interpreted as generated by the $e^{ \pm}$escaping from the PWNe system and inverse Compton scattering low-energy photons of the interstellar radiation fields.

Current source catalogs might be not complete and thus simulations of the pulsar populations are needed to extensively test the pulsar interpretation of the observed $e^{+}$flux. The idea of this work is to use the existing high-precision $e^{+}$data to constrain the main properties of the Galactic pulsar population and of the PWN acceleration needed to explain the observed cosmic-ray fluxes. We here simulate a large number of realizations for Galactic pulsar populations, comparing different updated models which reproduce ATNF catalog observations, instead of ad-hoc realization of pulsar characteristics. For each mock galaxy, we compute the resulting cosmic-ray $e^{+}$flux at the Earth from the PWN population and we fit it to the AMS-02 data to determine the physical parameters of these populations, and of individual sources, which are able to explain the observed positron flux. We also asses the impact of different assumptions on the radial distribution of sources, spin-down properties, propagation scenarios and positron emission properties. The remainder of the paper is organized as follows. In Sec. 2 our modeling for the $e^{ \pm}$production from a single PWN, and the basics of $e^{ \pm}$propagation in our Galaxy are illustrated. The strategies to simulate the Galactic pulsar populations and the different setups investigated are introduced in Sec. 3. The fit to AMS-02 data and the consequences for the pulsar characteristics of our minimization analysis are illustrated in Sec. 4, before concluding in Sec. 5

\section{Positrons from Galactic pulsars}

In this Section we illustrate our modeling for the $e^{ \pm}$production from a single PWN, while the strategies to simulate a Galactic pulsar population will be introduced in the next Section. We also briefly remind here the basics of $e^{ \pm}$propagation in our Galaxy.

\subsection{Injection of $e^{ \pm}$from pulsars}

Pulsars are rotating neutron stars with a strong surface magnetic field, and magnetic dipole radiation is believed to provide a good description for its observed loss of rotational energy. We consider a model in which $e^{ \pm}$are continuously injected at a rate that follows the pulsar spin-down energy. The injection spectrum $Q(E, t)$ of $e^{ \pm}$at a time $t$ is described as:

$$
Q(E, t)=L(t)\left(\frac{E}{E_{0}}\right)^{-\gamma_{e}} \exp \left(-\frac{E}{E_{c}}\right) \quad L(t)=\frac{L_{0}}{\left(1+\frac{t}{\tau_{0}}\right)^{\frac{n+1}{n-1}}}
$$


where the cut-off energy $E_{c}$ is fixed at $10^{5} \mathrm{TeV}, E_{0}$ is fixed at $1 \mathrm{GeV}, \gamma_{e}$ is the spectral index, $\tau_{0}$ is the characteristic time scale and $n$ defines the magnetic braking index. The total energy emitted by the source in $e^{ \pm}$is given by:

$$
E_{t o t}=\eta W_{0}=\int_{0}^{T} d t \int_{E_{1}}^{\infty} d E E Q(E, t)
$$

through which we obtain the value of $L_{0}$, fixing $E_{1}=0.1 \mathrm{GeV}$ ([4]). The parameter $\eta$ encodes the efficiency of conversion of the spin-down energy into $e^{ \pm}$pairs. $W_{0}$ is the initial rotational energy of a pulsar with a moment of inertia $I$ (typically assumed to be $10^{45} \mathrm{~g} \mathrm{~cm}^{2}$, as obtained from canonical neutron star values) and rotational frequency $\Omega_{0}=2 \pi / P_{0}$ :

$$
W_{0}=E_{\mathrm{rot}, 0}=\frac{1}{2} I \Omega_{0}^{2} .
$$

while the prediction on $\tau_{0}$ is derived to be:

$$
\tau_{0}=\frac{P_{0}}{(n-1) \dot{P}_{0}} .
$$

In our benchmark modeling we will consider only pulsars with ages above $20 \mathrm{kyr}$, since $e^{ \pm}$ accelerated to $\mathrm{TeV}$ energies in the termination shock are believed to be confined in the nebula or in the SNR until the merge of this system with the ISM, estimated to occur some kyr after the pulsar formation [5]. Our benchmark modeling thus considers possible effects of the release of $e^{ \pm}$ from the pulsar nebula, by leaving out sources for which the $e^{ \pm}$pairs might be still confined in the accelerator.

\subsection{Propagation of $e^{ \pm}$to the Earth}

Once charged particles are injected in the Galaxy, they can propagate and eventually reach the Earth. The $e^{ \pm}$number density per unit volume and energy $N_{e}(E, \mathbf{r}, t)$ of $e^{ \pm}$at an observed energy $E$, a position $\mathbf{r}$ in the Galaxy, and time $t$ is given by:

$$
N_{e}(E, \mathbf{r}, t)=\int_{0}^{t} d t^{\prime} \frac{b\left(E_{s}\right)}{b(E)} \frac{1}{\left(\pi \lambda^{2}\left(t^{\prime}, t, E\right)\right)^{\frac{3}{2}}} \times \exp \left(-\frac{\left|\mathbf{r}-\mathbf{r}_{\mathbf{s}}\right|^{2}}{\lambda\left(t^{\prime}, t, E\right)^{2}}\right) Q\left(E_{s}, t^{\prime}\right)
$$

where the integration over $t^{\prime}$ accounts for the PWN releasing $e^{ \pm}$continuously in time. The energy $E_{S}$ is the initial energy of $e^{ \pm}$that cool down to $E$ in a loss time $\Delta \tau$ :

$$
\Delta \tau \equiv \int_{E}^{E_{s}} \frac{d E^{\prime}}{b\left(E^{\prime}\right)}=t-t_{o b s} .
$$

The $b(E)$ term is the energy loss function, $\mathbf{r}_{\mathbf{s}}$ indicates the source position, and $\lambda$ is the typical propagation scale length defined as:

$$
\lambda^{2}=\lambda\left(E, E_{s}\right)^{2} \equiv 4 \int_{E}^{E_{s}} d E^{\prime} \frac{D\left(E^{\prime}\right)}{b\left(E^{\prime}\right)}=t-t_{o b s}
$$

where $D(E)$ is the diffusion coefficient. The flux of $e^{ \pm}$at the Earth for a source of age $T$ and distance $d$ is given then by:

$$
\Phi_{e^{ \pm}}(E)=\frac{c}{4 \pi} \mathcal{N}_{e}(E, r=d, t=T)
$$




\begin{tabular}{c|c|c|c}
\hline \hline $\begin{array}{c}\text { PSR } \\
\text { property }\end{array}$ & $\begin{array}{c}\text { Simulated } \\
\text { quantity }\end{array}$ & Benchmark & Variations \\
\hline Age & $T$ & Uniform $\left[0, t_{\max }\right]$ & - \\
\hline & $P_{0}$ & CB20[11] & FK06[12] \\
Gaussian [0.3s; 0.15s] & - \\
& $\log 10(\mathrm{~B})$ & $\begin{array}{c}\text { Gaussian [12.85G;0.55G] } \\
\text { Uniform [2.5-3] }\end{array}$ & $\begin{array}{c}\text { Gaussian [12.65G; 0.55G] } \\
\text { Constant [3] }\end{array}$ \\
& $\mathrm{n}$ & Uniform [0-1] & Constant [0] \\
\hline$e^{ \pm}$injection & $\gamma_{e}$ & Uniform [1.4-2.2] & - \\
& $\eta$ & Uniform [0.01-0.1] & - \\
\hline Radial & $\mathbf{r}$ & $\rho_{L}(r)[13]$ & $\rho_{F}(r)[12]$ \\
distribution & & & \\
\hline \hline
\end{tabular}

Table 1: Summary of the quantities from which we build the mock pulsar catalogues. We report the distributions followed in the simulation of these parameters in our benchmark case, as well as the tested variations. See Sec. 3 for details.

In our analysis we will consider as benchmark case the propagation parameters as derived in [6] from a fit to the latest AMS-02 data for the B/C, antiprotons and proton data. We will label this model as Benchmark-prop. In this model the diffusion coefficient is parametrized as a power-law $D(E)=D_{0} \mathcal{R}^{\delta}$ where $D_{0}=0.042 \mathrm{kpc}^{2} / \mathrm{Myr}, \delta=0.459$. The value of $L$ is unconstrained and fixed to $4 \mathrm{kpc}$, which is compatible with the recent results of Ref. [7]. Energy losses are computed on the interstellar photon populations at different wavelengths as in [8], and on the Galactic magnetic field with intensity $B=3 \mu \mathrm{G}$. As a comparison, we will also implement the SLIM-MED model derived in [9], with the interstellar photon populations taken from [10] and $B=1 \mu \mathrm{G}$. This model assumes $D_{0}=0.036 \mathrm{kpc}^{2} / \mathrm{Myr}, \delta=0.499$ and $L=4.67 \mathrm{kpc}$. For the $S L I M-M E D$ model, the flux coming from a single source is smoother with respect to the one obtained with the Benchmark-prop.

\section{Simulations of Galactic pulsar populations}

We simulate catalogs of Galactic pulsars, following the injection and propagation modeling described in Sec. 2. For each realization of a Galactic population, we compute the $e^{+}$flux from each pulsar. In all the simulations, the total number of sources is fixed at $N_{\mathrm{PSR}}=t_{\max } \dot{N}_{P S R}$, where $t_{\max }$ is the maximum simulated age and $\dot{N}_{P S R}$ is the pulsar birth rate. Different estimates for Galactic $\dot{N}_{P S R}$ range from one to four per century [13]. We here assume the maximum age of the sources to be $t_{\text {max }}=10^{8} \mathrm{yr}$, and $\dot{N}_{P S R}=0.01 \mathrm{yr}^{-1}$. In order to compute the $e^{+}$flux at the Earth for each mock source, we need a prediction for its position in the Galaxy, its age and the source term $Q(E, t)$ (see Eq. 1). Specifically, the fundamental parameters of each simulation are: the age of the source $T$, $P_{0}, B, n, \alpha, \gamma_{e}, \eta$ and the position $\mathbf{r}$ in Galactocentric coordinates. A summary of the simulated quantities is illustrated in Tab. 1 and outlined in what follows.

First of all, the simulation assigns to each mock pulsar a certain age $T$ extracted uniformly between $t=0$ and $t_{\max }$. Then we sample the values of $P_{0}, B, n$ and $\alpha$ from the distributions provided in [11], which will be our benchmark model (CB20). Specifically, $P_{0}$ is simulated according to a 
Gaussian distribution with $P_{0, \text { mean }}=0.3 \mathrm{~s}$ and $P_{0, s t d}=0.15 \mathrm{~s}$. We also impose a lower bound on $P_{0}=0.83 \mathrm{~ms}$, as physically motivated in [14]. The magnetic field is simulated following a Gaussian distribution for $\log _{10}(B)$, with $\log _{10}(B)_{\text {mean }}=12.85 \mathrm{G}$ and $\log _{10}(B)_{s t d}=0.55 \mathrm{G}$. The values of $n$ and $\cos \alpha$ are taken from uniform distributions, respectively in the range [2.5-3] and [0,1]. We note that from the simulated values of $P_{0}, B, n, \alpha$ we derive for each pulsar $W_{0}$ and $\tau_{0}$, through Eqs. 3 and 4 . Since the spectral index $\gamma_{e}$ of accelerated particles is uncertain, and may vary significantly for each PWNe [2], it is sampled from uniform distributions within [1.4-2.2]. Finally, the value of $\eta$ for each source is sampled from a uniform distribution in the range [0.01-0.1]. In order to assess the effects of different distributions for $P_{0}, B, n, \alpha$, we consider the alternative model in [12] (FK06 hereafter) and reported in Tab. 1.

\subsection{Spatial distribution of pulsars in the Galaxy}

To complete the construction of the mocked catalogues of Galactic sources the position $\mathbf{r}$ of each pulsar has to be determined. Using gammapy.astro.population [15] we sample $\mathbf{r}$ for each source adopting the radial surface density of pulsars $\rho_{L}(r)$ proposed by [13](see Eq. 9, left). As a comparison, we will also consider the radial surface density $\rho_{F}(r)$ in [12](see Eq. 9, right):

$$
\rho_{L}(r)=A_{1}\left(\frac{r}{r_{\odot}}\right) \exp \left[-C\left(\frac{r-r_{\odot}}{r_{\odot}}\right)\right] \quad \rho_{F}(r)=A_{2} \frac{1}{\sqrt{2 \pi} \sigma} \exp \left(-\frac{\left(r-r_{\odot}\right)^{2}}{2 \sigma^{2}}\right) .
$$

The position $\mathbf{r}$ is fully determined by accounting for the spiral arm structure of the Milky Way according to the model of Ref. [12] (see their Table 2 for the spiral arm parameters). The $\rho_{F}(r)$ distribution increases the number of sources in the two spiral arms beside the Earth with respect to $\rho_{L}(r)$. The distance of each source is $d=\left|\mathbf{r}-\mathbf{r}_{\odot}\right|$, with $\mathbf{r}_{\odot}=(8.5 \mathrm{kpc}, 0,0)$.

\subsection{Summary of simulation setups}

In this section we recap the combinations of the different simulation setups described before and listed in Tab. 1.

ModA (benchmark). Spin-down and pulsar evolution properties are taken from CB20 [11], while the radial distribution of sources is modelled with $\rho_{L}(r) . \eta$ and $\gamma_{e}$ are extracted from uniform distributions reported in Tab. 1, while the propagation in the Galaxy is taking into account with Benchmark-prop following Ref. [6].

ModB (radial distribution effect). Same as ModA but with the radial surface density of sources $\rho_{F}(r)$ instead of $\rho_{L}(r)$ (see Eq. 9, [12]).

ModC (spin-down properties effect). Same as ModA, but spin-down properties are taken from FK06 [12].

ModD (propagation effect). Same as ModA apart for propagation in the Galaxy, modelled as in Ref. [9] (their model SLIM-MED). 


\begin{tabular}{|c|c|c|c|}
\hline & $\chi_{\text {red }}^{2}<2$ & $\chi_{\text {red }}^{2}<1.5$ & $\chi_{\text {red }}^{2}<1$ \\
\hline ModA & 15. & 8 & 4 \\
ModB & 30. & 19 & 6 \\
ModC & 15. & 10 & 3 \\
ModD & 42. & 25 & 10 \\
\hline
\end{tabular}

Table 2: Number of simulations (out of 1000) that produce a $\chi_{\text {red }}^{2}$ smaller than a $1,1.5$ or 1 , in the fit to AMS-02 data [1], for each simulation setup.

\section{Results}

We describe here how we performed the fit to the data, and which are the physics results of our minimization analysis. For each simulation setup described in Sec. 3.2, we build and test 1000 simulations. We compute the $e^{+}$flux at the Earth as the sum of the primary component due to pulsar emission (see Sect. 2 and Sect. 3), and a secondary component due to the fragmentation of cosmic rays on the nuclei of the ISM, taken from [6] and [9] consistently with the propagation model employed. The secondary component enters in our fits with a free normalization factor $A_{S}$, which we generously let to vary between 0.01 and 3 . We also let the total flux generated by all pulsars to be shifted by an overall normalization faction $A_{P}$. We fit AMS-02 data [1] above 10 $\mathrm{GeV}$, in order to avoid strong influence from solar modulation and other possible low energy effects [16]. Nevertheless, we correct our predictions for solar modulation effects following the force field approximation and leaving the Fisk potential $\phi$ free to vary between 0.4 and $1.2 \mathrm{GV}$. The comparison of our predictions with the AMS-02 data is performed by a standard $\chi^{2}$ minimization procedure.

\subsection{Comparison to the AMS-02 $e^{+}$data}

The comparison through a fit of the predictions for the total $e^{+}$flux to the AMS-02 data is performed for all the 1000 simulations built for each scenario A-B-C-D. In Tab. 2 we report the number of simulations, out of 1000, that produce different values of $\chi^{2} /$ d.o.f. $=\chi_{\text {red }}^{2}$ for each simulation setup. In all the tested setups, the number of mock galaxies with a $\chi_{\text {red }}^{2}<1$ (2) does not exceed $1 \%(4 \%)$. We present here some preliminary results for the total $e^{+}$flux in our benchmark setup ModA. In Fig. 1 we plot the $e^{+}$flux obtained for two illustrative simulated galaxies with $\chi_{\text {red }}^{2}<1$. The contributions from each pulsar, from the secondary emission and their sum are shown along with the AMS-02 data. All the good fits to the data find a value for $A_{S}$ between 2 and 2.5, which might at least partially ascribable to an underestimation of spallation cross sections. The difference between ModA and ModD is relative only to the propagation and the energy losses modeling. ModD promotes a higher number of simulations to be compatible with the data: the SLIM-MED model produces fluxes from a single source which are smoother with respect to Benchmark-prop. Concerning the other simulation setups analysed, we do not find significant differences between ModA and ModC, and so between CB20 and FK06 pulsar evolution models. On the other hand, ModB promotes a higher number of simulations to be compatible with the data: since the $\rho_{F}(r)$ radial distribution predicts the presence of a higher number of sources in the spiral arms beside the Earth with respect to $\rho_{L}(r)$, for ModB there is a higher probability to simulate sources 
close to the Earth with characteristics compatible with the AMS-02 data. As for the allowed overall

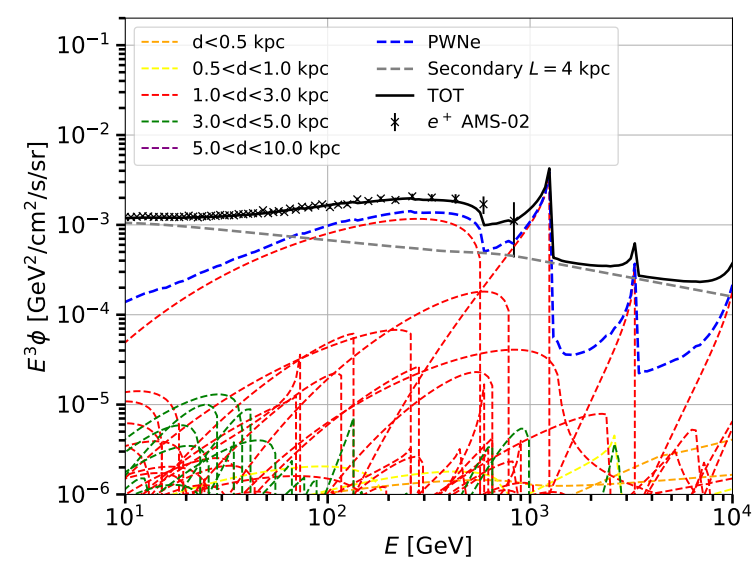

(a)

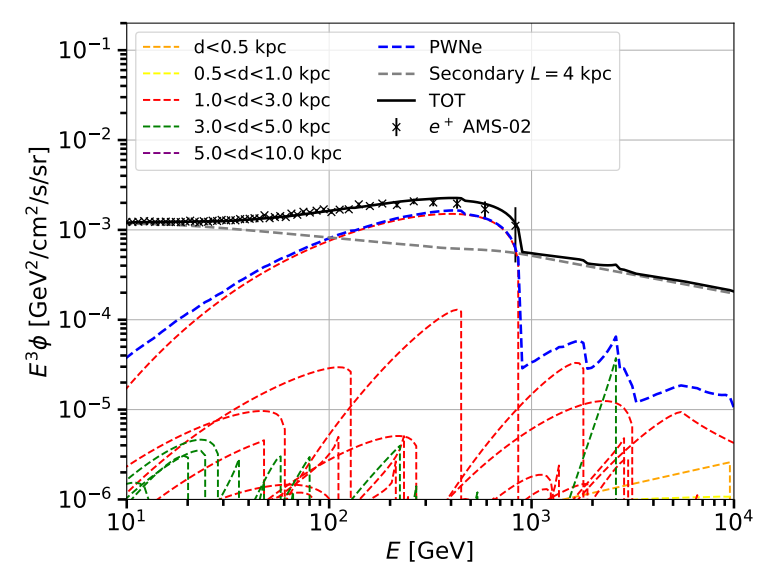

(b)

Figure 1: Comparison between the AMS-02 $e^{+}$flux data [1] (black points) and the flux from secondary production (grey dashed line) and pulsars (blue dashed line) for two ModA realizations of the Galaxy with $\chi_{\text {red }}^{2}<1$. The contributions from each pulsar (reported with different colors depending on their distance from the Earth) are shown.

normalization $A_{P}$ of the pulsars' primary flux, we find on average values slightly smaller than one. In these particular cases, the dominant contribution comes from source in the distance ring between 1 and $3 \mathrm{kpc}$.

\section{Conclusions}

In this work we have performed several fits to the AMS-02 $e^{+}$flux data testing a variety of simulated pulsar population. The novelty of this paper is to use the existing high-precision $e^{+}$data to constrain the main properties of the Galactic pulsar population and of the PWN acceleration needed to explain the observed cosmic-ray fluxes. We have simulated a large number of realizations for Galactic pulsar populations, comparing different updated models built on parameter distributions calibrated on observations, instead of ad-hoc realizations of pulsar characteristics. We used the AMS-02 data to determine the physical parameters of these populations, and of individual sources, which are able to explain the observed positron flux. We investigated the impact of different assumptions on the radial distribution of sources, spin-down properties, propagation scenarios and positron emission properties. Only a few galaxies for each setup are compatible with the data.

\section{Bibliography}

[1] AMS Collaboration Collaboration, M. Aguilar, L. Ali Cavasonza, G. Ambrosi, et al., Towards understanding the origin of cosmic-ray positrons, Phys. Rev. Lett. 122 (Jan, 2019) 041102.

[2] D. Malyshev, I. Cholis, and J. Gelfand, Pulsars versus Dark Matter Interpretation of ATIC/PAMELA, Phys. Rev. D80 (2009) 063005, [arXiv: 0903.1310]. 
[3] HAWC Collaboration, A. U. Abeysekara et al., Multiple Galactic Sources with Emission Above 56 TeV Detected by HAWC, Phys. Rev. Lett. 124 (2020), no. 2021102 , [arXiv: 1909.0860].

[4] I. Sushch and B. Hnatyk, Modelling of the radio emission from the Vela supernova remnant, Astron. Astrophys. 561 (2014) A139, [arXiv: 1312.0777].

[5] P. Blasi and E. Amato, Positrons from pulsar winds, Astrophysics and Space Science Proceedings 21 (2011) 624, [arXiv: 1007 . 4745].

[6] M. D. Mauro and M. W. Winkler, Multimessenger constraints on the dark matter interpretation of the fermi-lat galactic center excess, arXiv: 2101.1102.

[7] N. Weinrich, M. Boudaud, L. Derome, Y. Genolini, J. Lavalle, D. Maurin, P. Salati, P. Serpico, and G. Weymann-Despres, Galactic halo size in the light of recent AMS-02 data, Astron. Astrophys. 639 (2020) A74, [arXiv: 2004 . 0044].

[8] S. Vernetto and P. Lipari, Absorption of very high energy gamma rays in the Milky Way, Phys. Rev. D94 (2016), no. 6 063009, [arXiv: 1608.0158].

[9] Y. Génolini, M. Boudaud, M. Cirelli, L. Derome, J. Lavalle, D. Maurin, P. Salati, and $\mathrm{N}$. Weinrich, New minimal, median, and maximal propagation models for dark matter searches with galactic cosmic rays, arXiv:2103.0410.

[10] T. Delahaye, J. Lavalle, R. Lineros, F. Donato, and N. Fornengo, Galactic electrons and positrons at the Earth: new estimate of the primary and secondary fluxes, A\&A $\mathbf{5 2 4}$ (Dec., 2010) A51, [arXiv: 1002 . 1910].

[11] A. Chakraborty and M. Bagchi, Understanding the Galactic population of normal pulsars: A leap forward, arXiv:2012.1324.

[12] C.-A. Faucher-Giguere and V. M. Kaspi, Birth and Evolution of Isolated Radio Pulsars, ApJ 643 (May, 2006) 332-355, [astro-ph/0512585].

[13] D. R. Lorimer, The Galactic Population and Birth Rate of Radio Pulsars, astro-ph/0308501.

[14] J. Lattimer and M. Prakash, Neutron star observations: Prognosis for equation of state constraints, Physics Reports 442 (Apr, 2007) 109-165.

[15] A. Donath and others., Gammapy: An open-source Python package for gamma-ray astronomy, in 34th International Cosmic Ray Conference (ICRC2015), vol. 34, p. 789, Jul, 2015. arXiv: 1509.0740.

[16] M. Boudaud, E. F. Bueno, S. Caroff, Y. Genolini, V. Poulin, V. Poireau, A. Putze, S. Rosier, P. Salati, and M. Vecchi, The pinching method for Galactic cosmic ray positrons: implications in the light of precision measurements, Astron. Astrophys. 605 (2017) A17, [arXiv: 1612.0392]. 